\title{
In Vivo Tumor Transfection with Superantigen plus Cytokine Genes Induces Tumor Regression and Prolongs Survival in Dogs with Malignant Melanoma
}

\author{
Steven W. Dow, ${ }^{\star}$ Robyn E. Elmslie, ${ }^{\S}$ Andrew P. Willson, ${ }^{*}$ Lisa Roche, $\|$ Cori Gorman, \\ *Division of Basic Immunology, Department of Medicine, National Jewish Medical and Research Center, Denver, Colorado 80206; \\ ${ }^{\ddagger}$ Department of Immunology and the Cancer Center, University of Colorado Health Sciences Center, Denver, Colorado 80206; ${ }^{\S}$ Veterinary \\ Cancer Specialists, Denver, Colorado 80210; and ${ }^{\|}$Megabios Corporation, Burlingame, California 94010
}

\begin{abstract}
In vivo transfection of established tumors with immunostimulatory genes can elicit antitumor immunity. Therefore, we evaluated the safety and efficacy of intratumoral injections of a bacterial superantigen with a cytokine gene in dogs with malignant melanoma, a spontaneous and highly malignant canine tumor. 26 dogs with melanoma were treated with lipid-complexed plasmid DNA encoding staphylococcal enterotoxin B and either GM-CSF or IL-2. Dogs were evaluated for treatment-associated toxicity, tumor responses, immunologic responses, and survival times. The overall response rate (complete or partial remissions) for all 26 dogs was $46 \%$ (12 of 26), and was highest in patients with smaller tumors. Toxicity was minimal or absent in all dogs. Injected tumors developed marked infiltrates of CD4 + and $\mathrm{CD} 8+\mathrm{T}$ cells and macrophages, and tumor regression was associated with development of high levels of antitumor cytotoxic T lymphocyte activity in peripheral blood lymphocytes. Survival times for animals with stage III melanomas treated by intratumoral gene therapy were prolonged significantly compared with animals treated with surgical tumor excision only. Thus, local tumor transfection with superantigen and cytokine genes was capable of inducing both local and systemic antitumor immunity in an outbred animal with a spontaneously developing malignant tumor. (J. Clin. Invest. 1998. 101:2406-2414.) Key words: melanoma • staphylococcal enterotoxin B - granulocyte macrophage colonystimulating factor • interleukin 2 - lymphocytes
\end{abstract}

\section{Introduction}

A variety of immunologic strategies have been used to elicit antitumor responses of sufficient magnitude to cause regression of established tumors. These approaches generally fall into one of two categories: immunization with genetically modified autologous tumors cells (tumor vaccines), or induc-

Address correspondence to Dr. Terry A. Potter, Division of Basic Immunology, National Jewish Medical and Research Center, 1400 Jackson Street, Denver, CO 80206. Phone: 303-398-1305; FAX: 303398-1396. L. Roche's current address is GenPharm International, San Jose, CA 95131. C. Gorman's current address is DNA Bridges, Inc., San Francisco, CA 94020.

Received for publication 29 April 1997 and accepted in revised form 19 March 1998.

J. Clin. Invest.

(C) The American Society for Clinical Investigation, Inc. 0021-9738/98/06/2406/09 \$2.00

Volume 101, Number 11, June 1998, 2406-2414

http://www.jci.org tion of immune responses against specific tumor antigens (1-15). Autologous tumor vaccines, though promising, may be limited in their clinical applicability by the requirement for establishing tumor lines for each patient and by the need for efficient ex vivo transfection (1-9). Antigen-specific immunization requires identification of specific tumor antigens and assumes that all or most tumor cells present in an individual express those antigens (10-15). The extreme polymorphism of human MHC molecules may also limit the general applicability of tumor antigen- or tumor peptide-specific approaches.

Direct in vivo transfection of tumors is an alternative strategy that offers several advantages. Such an approach, using immunostimulatory genes, may induce immune responses against a broader array of tumor antigens than immunization with in vitro-cultured tumor cells or with dendritic cells. Nabel and co-workers first reported use of intratumoral injection of plasmid DNA complexed to cationic lipids to elicit antitumor responses in mice; a similar approach was evaluated and found to be safe in a pilot study of humans with melanoma $(16,17)$. Repeated induction of a sufficiently potent local immune stimulus by direct gene transfer may also be sufficient to break the state of relative immune tolerance that develops to self-antigens expressed by established tumors (18). More recently, gene gun-mediated tumor transfection with the IL-12 gene was reported to induce impressive tumor regressions in several murine tumor models, and a similar approach using the IL-6 gene was also reported to slow tumor growth in vivo $(19,20)$.

$\mathrm{T}$ cells activated by bacterial superantigens (SAgs) ${ }^{1}$ proliferate, secrete cytokines (e.g., IFN- $\gamma$, TNF- $\alpha$, IL-2, and IL-12), develop strong cytolytic activity, and when adoptively transferred can mediate tumor regression (21-27). In vivo therapy with the staphylococcus enterotoxin A protein conjugated to a tumor-specific $\mathrm{mAb}$ has also been reported to induce $\mathrm{T}$ cell infiltration and tumor regression in mice (28). We reported recently that expression of SAg genes in mouse tissues in vivo elicited pronounced localized mononuclear cell inflammatory responses (29). Further, we found that intratumoral injection of SAg genes could induce regression of experimental tumors in mice, and that the antitumor effect was augmented by combination with a cytokine gene. ${ }^{2}$ Bacterial SAgs are more potent $\mathrm{T}$ cell-activating agents in dogs and humans than in mice, therefore making it likely that their local immune-stimulatory effects after in vivo gene transfer would also be greater (30).

Therefore, we conducted a pilot study to assess the safety,

1. Abbreviations used in this paper: CTL, cytotoxic T lymphocyte; DOPE, dioleoyl phosphatidylethanolamine; DOTMA, $N$-[1-(2,3-dioleyloxy)propyl]- $N, N, N$-triethylammonium; SAg, superantigen; SEB, staphylococcal enterotoxin B.

2. Dow, S.W., P.M. Walsh, D. Kummer, L. Roche, C. Gorman, and T.A. Potter, manuscript submitted for publication. 
efficacy, and immunologic effects of direct in vivo tumor transfection with the staphylococcus enterotoxin B (SEB) gene and a cytokine gene (either GM-CSF or IL-2) in a clinically relevant, large animal model of melanoma. Canine melanoma is a spontaneous, highly aggressive tumor of dogs that metastasizes readily to the lungs, which is the primary cause of death for most canine melanoma patients $(31,32)$. We found that direct intratumoral injection of SEB and cytokine DNA in dogs induced local antitumor immunity, as evidenced by tumor regression and the development of pronounced infiltration of $\mathrm{T}$ cells and macrophages. Furthermore, local tumor injection also induced systemic antitumor immunity, as evidenced by the presence of high levels of antitumor cytotoxic T lymphocyte (CTL) activity in the bloodstream of some responding patients and by the significantly prolonged survival times observed in treated animals with advanced tumors. Despite the known toxicity of SAgs in dogs, systemic toxicity was not observed in any treated animals.

\section{Methods}

Cloning and expression of SEB, canine GM-CSF, and canine IL-2. The gene for SEB was cloned and expressed in the eukaryotic expression plasmid PCR3 (Invitrogen Corp., San Diego, CA), as reported previously (29). The genes for canine GM-CSF and IL-2 were cloned by PCR from cDNA prepared from normal, mitogen-stimulated canine PBMC, using primers based on the published canine GM-CSF and IL-2 sequences (GenBank accession nos. S49738 and D30710, respectively). The genes were cloned into PCR3 and sequenced, and their biologic activity was confirmed by bioassays (data not shown).

Autologous tumor cultures. Primary autologous melanoma cultures were established from pretreatment biopsies obtained from dogs with oral melanoma. Biopsy tissues were minced and digested with $0.02 \%$ collagenase (Sigma Chemical Co., St. Louis, MO), and the dissociated cells were seeded in 24-well plates in Eagle's modified essential medium supplemented with $10 \%$ FBS, pyruvate, nonessential amino acids, glutamine, and antibiotics. Wells with colonies of tumor cells without contaminating fibroblasts were selected and expanded to establish autologous melanoma lines. Autologous tumor lines were classified as melanomas based on several criteria, including morphology, the presence in some tumors of melanin granules, expression (by immunocytochemistry) of S-100 protein, and by reverse transcription PCR for expression of melanoma antigen recognized by T cells (MART-1) mRNA (data not shown).

In vitro transfection of melanoma cells. Melanoma cells from four different canine melanoma primary cultures were transfected with plasmid DNA encoding either SEB or empty PCR3 vector (mock), using Lipofectamine (GIBCO BRL, Gaithersburg, MD). After transfection, cells were washed, refed with fresh medium, and cultured for an additional $48 \mathrm{~h}$. The supernatants were then harvested, and the adherent cells were washed, lysed by three repeated freeze-thaw cycles, centrifuged, and frozen.

Lymphocyte proliferation assay for SEB activity. SAg biological activity was assessed by measuring the lymphocyte-stimulatory activity present in supernatants or lysates of transfected melanoma cells, as described previously except that normal canine PBMC were used instead of human PBMC (29). Lymphocyte proliferation was assayed after $72 \mathrm{~h}$ in culture, using $\left[{ }^{3} \mathrm{H}\right]$ thymidine incorporation. Results are expressed as the mean $( \pm \mathrm{SD})$ incorporated ${ }^{3} \mathrm{H}$ activity in counts per minute. Negative controls included mock-transfected tumor cells, and purified SEB (Toxin Technology, Inc., Sarasota, FL) was used as a positive control.

SEB antigen ELISA. An antigen capture ELISA was used to quantitate production of SEB protein by in vitro-transfected tumor cells, as described previously (29). Briefly, supernatants and lysates from in vitro-transfected canine melanoma cultures (see above) were diluted and assayed in triplicate. The amount of SEB present was quantitated by comparison with a standard curve generated with purified SEB. Data were plotted as the mean $( \pm S D)$ of triplicate SEB determinations.

DNA and liposome preparation. Plasmid DNA for injection was prepared by modified alkaline lysis and cesium chloride gradient centrifugation. The plasmid DNA was resuspended to a final concentration of $1.0 \mathrm{mg} / \mathrm{ml}$ in sterile PBS. Liposomes for injection were prepared by combining equimolar amounts of DOTMA $(N-[1-(2,3-$ dioleyloxy)propyl]- $N, N, N$-triethylammonium; Syntex Corp., Palo Alto, CA) and DOPE (dioleoyl phosphatidylethanolamine; Avanti Polar Lipids, Inc., Alabaster, AL). The lipids were evaporated to dryness in a vacuum desiccator, and liposomes were prepared by reconstitution in sterile PBS to a final concentration of $1.0 \mathrm{mg} / \mathrm{ml}$, followed by sonication for $7 \mathrm{~min}$, as described previously (33). Before injection, equal volumes of liposomes and plasmid DNA were mixed and allowed to complex at room temperature for $30 \mathrm{~min}$. At the time of injection, the liposome/DNA mixture was further diluted in balanced salt solution to a final volume of 1-2 $\mathrm{ml}$, depending on tumor size.

A second cationic lipid formulation was also used to treat six dogs in this study because this formulation had shown superior in vivo tumor transfection efficiencies compared with DOTMA/DOPE and other cationic lipids (Gorman, C., unpublished data). These six dogs had been unsuccessfully treated initially using DNA complexed to the DOTMA/DOPE lipids. This second lipid was comprised of DOTIM (octadecenoyloxy[ethyl-2-heptadecenyl-3-hydroxyethyl] imidazolinium chloride) and cholesterol, which was complexed to plasmid DNA in a 1:1 ratio (micrograms of DNA to nanomoles of liposome) (34).

Immunohistochemical analysis of SEB gene expression in tumor biopsies. Pretreatment and $48 \mathrm{~h}$ postinjection tumor biopsies were obtained from five different canine patients with melanoma. Tumor biopsies were snap frozen and cryosectioned to a thickness of $4.0 \mu \mathrm{m}$. An SEB-specific affinity-purified rabbit antiserum (Toxin Technology, Inc.) was used for detection of SEB expression. Controls included posttreatment tumor biopsies reacted with an irrelevant rabbit antiserum and pretreatment tumor biopsies reacted with anti-SEB antisera. Tissue sections were fixed in acetone, then incubated with appropriately diluted primary antisera followed by biotinylated goat anti-rabbit antiserum (Kirkegaard \& Perry Laboratories, Inc., Gaithersburg, MD), followed by avidin-biotin-horseradish peroxidase complex (Vector Laboratories, Inc., Burlingame, CA). Slides were developed using diaminobenzidine as a substrate, then were lightly counterstained with hematoxylin and coverslipped.

Immunocytochemical analysis of tumor-infiltrating leukocytes. A panel of $\mathrm{mAbs}$ developed against canine leukocyte antigens, including anti-canine CD4, anti-canine CD8 ( $\alpha$ chain), anti-canine CD11b, and anti-canine CD11c (provided by Dr. Peter Moore, Leukocyte Antigen Biology Laboratory, University of California, Davis) was used to identify tumor-infiltrating leukocytes in tumor biopsies (35). Biopsies were processed and immunostained essentially as described for detection of SEB expression, with the substitution of a biotinylated anti-mouse IgG reagent for the anti-rabbit secondary antibody.

CTL assays. Fresh PBMC were obtained from dogs at various times during treatment and were restimulated in vitro for $6 \mathrm{~d}$ with irradiated (6,000 rads) autologous tumor cells at a ratio of 40:1 in 12well plates. On day 2 in culture, recombinant human IL-2 was added to a final concentration of $0.5 \mathrm{ng} / \mathrm{ml}$. For CTL assay, decreasing numbers of effector cells, beginning at an $\mathrm{E} / \mathrm{T}$ ratio of 50:1, were added in $100 \mu \mathrm{l}$ media to duplicate wells of Linbro plates. Tumor target cells were labeled with ${ }^{51} \mathrm{Na}$-chromate (New England Nuclear, Medford, $\mathrm{MA})$ at $37^{\circ} \mathrm{C}$ for $1.5 \mathrm{~h}$, and $5 \times 10^{3}$ target cells were added per well. The plates were incubated at $37^{\circ} \mathrm{C}$ for $4 \mathrm{~h}$, and $\mathrm{Cr}$ release in supernatants was quantitated by $\gamma$ counter. The percentage of specific lysis was determined according to the following formula: [(observed release - spontaneous release)/(spontaneous release - maximal release) $] \times 100$. CTL assays were done using both autologous mela- 
noma target cells and allogeneic canine melanoma target cells to assess the MHC specificity of target cell killing. The data presented are representative of at least two CTL assays per patient. Canine melanoma lines resistant to $\mathrm{T}$ cell cytotoxicity were not observed (data not shown).

$P B M C$ proliferative responses to purified SEB protein. The ability of PBMC from SEB-treated and control dogs to proliferate to 500 $\mathrm{ng} / \mathrm{ml}$ purified SEB was assessed using a thymidine incorporation assay as described above for detection of SEB activity in transfected cells. Results were expressed as stimulation indices, which were calculated from the mean incorporated ${ }^{3} \mathrm{H}$ activity (in cpm) of wells with and without SEB, according to the following formula: stimulation in$\operatorname{dex}=($ SEB-stimulated proliferation - background proliferation $) /$ background proliferation.

$S E B$ antibody ELISA. Antibodies to SEB were assayed in the serum of treated and control dogs using an ELISA assay. Briefly, immunoassay plates (Immunolon; Dynatech Laboratories, Inc., Chantilly, VA) were coated with purified anti-SEB mAb (kindly provided by Dr. John Kappler, National Jewish Medical and Research Center), blocked with nonfat dry milk, and incubated with $100 \mathrm{ng} / \mathrm{ml}$ purified SEB protein, then dog serum samples diluted 1:1,000 in PBS were added, followed by goat anti-dog IgG (Kirkegaard \& Perry Laboratories, Inc.) and then streptavidin-horseradish peroxidase (Zymed Laboratories, Inc., South San Francisco, CA); the plates were then developed by addition of 2,2'-azinobis-(3-ethylbenzthiazoline-6-sulfonate) substrate, and OD was determined by an automated ELISA reader. Serum from 8 treated animals and 10 control dogs was evaluated for detection of anti-SEB antibodies.

Treatment and monitoring of dogs. Dogs entered into the study had histologic confirmation of melanoma; the tumors were staged by a veterinary oncologist, and the dogs were determined to be free of severe underlying systemic illnesses. During the study, dogs did not receive radiation or chemotherapy or any form of potentially immunosuppressive medication. The study protocol was approved by the Institutional Animal Care and Use Committee; the dogs' owners were advised of the experimental nature of the treatment, and all owners granted written informed consent to treat. During treatment, dogs were monitored for changes in attitude, appetite, body temperature, body weight, and tumor appearance. Body temperature was monitored daily for the first $72 \mathrm{~h}$ after the first injection. Complete blood counts and serum biochemical analyses were done for each dog once monthly during the initial $12 \mathrm{wk}$ of the study. Tumor responses were measured by sequential photography and measurement of tumor dimensions. Tumor responses were classified as PD (progressive disease; $>50 \%$ increase in tumor size), SD (stable disease; $<50 \%$ increase in tumor size), PR (partial remission; $>50 \%$ reduction in tumor size), or CR (complete remission; tumor no longer detectable). Thoracic radiographs were done before treatment and at 6 and $12 \mathrm{wk}$ of the study, and at longer intervals in long-term surviving animals.
The trial was designed as an initial 12-wk study, with animals treated every 2 wk by direct intratumoral injection of plasmid DNA complexed to cationic liposomes. Two different doses of plasmid DNA were injected, based on tumor size: $400 \mu \mathrm{g}$ total plasmid DNA was injected for tumors $<18 \mathrm{~cm}^{3}, 800 \mu \mathrm{g}$ total plasmid DNA for tumors $>18 \mathrm{~cm}^{3}$. Equal amounts of SEB and GM-CSF (or IL-2) plasmid DNA were mixed and injected together. These doses were found to be nontoxic in preliminary dose escalation studies done in two dogs (data not shown). At each treatment, dogs were anesthetized briefly with a short-acting anesthetic. The tumor was injected in multiple sites (both intratumoral and peritumoral) using a syringe and 25gauge needle. In addition, when possible, the nearest tumor-draining lymph node was also injected. For all animals whose tumors underwent partial regression or in those where tumor size remained stable during the 12-wk induction trial, additional follow-up treatment was administered at the same DNA dose but given at once-monthly intervals. Treatments were continued until complete remission was achieved, at which time DNA injections ceased. Animals with progressive tumors were killed humanely.

Statistical analysis. Survival times between control and treated stage III melanoma dogs were compared by both log-rank analysis and Wilcoxon tests. Significant differences were established for $P<$ 0.05 .

\section{Results}

Melanoma cells transfected with SEB in vitro produce biologically active $S E B$ protein intracellularly and extracellularly. Four different canine melanoma cell lines were transfected in vitro with plasmid DNA encoding either SEB or noncoding plasmid (empty vector). Both supernatants and cell lysates harvested $48 \mathrm{~h}$ after transfection contained SEB protein, as detected by SEB-specific ELISA (Fig. $1 A$ ). SEB could be detected in both supernatants and lysates of all four transfected melanoma lines, but the amount of SEB produced by different melanoma lines varied widely, despite being transfected with an equivalent amount of plasmid DNA on a per cell basis (Fig. 1). This variability may have resulted from cell-specific differences in transfection efficiency, or from differences in the ability of different melanoma cells to produce and export SAgs.

The SEB produced by transfected melanoma cells was also biologically active, as evidenced by the strong proliferative response of normal canine lymphocytes to both supernatants and lysates from SEB-transfected cells (Fig. $1 B$ ). After liposome-mediated transfection in vitro, between 1 and $10 \%$ of cells expressed SEB antigen, depending on the particular cell

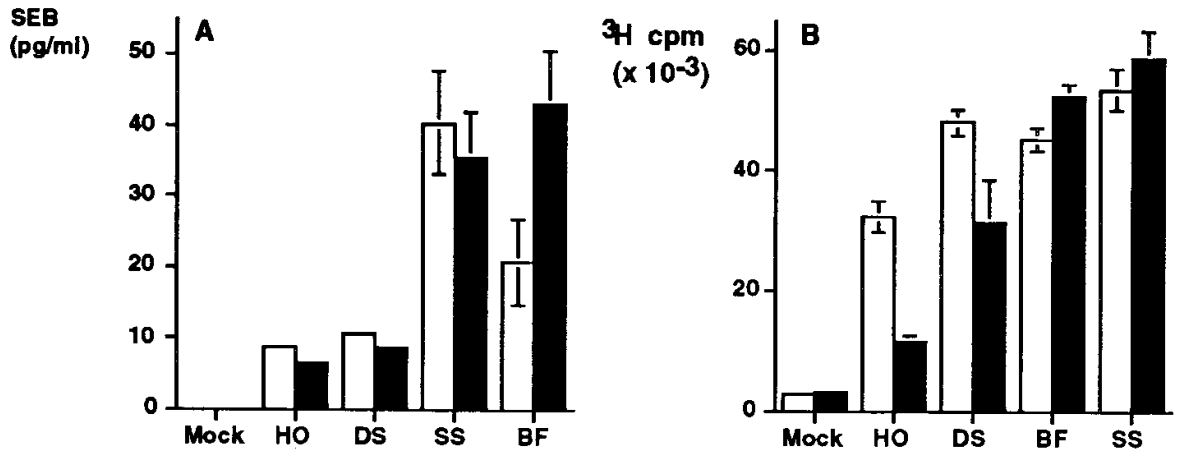

Figure 1. Production of biologically active SEB by melanoma cells transfected in vitro. Four canine melanoma cell lines established from four dogs in this study (identified as $B S, H O, S S$, and $S W$ ) were transfected in triplicate in vitro for $48 \mathrm{~h}$ with plasmid DNA encoding SEB or with an empty vector (Mock). Supernatants and cell lysates were harvested, and the SEB concentration was quantitated by antigen ELISA. The mean $( \pm$ SD) SEB concentration in supernatants (white bars) and cell lysates (black bars) for each cell line were plotted $(A)$. Supernatants (white bars) and

lysates (black bars) were also assayed for lymphocyte proliferative activity, using normal canine PBMC (B). After $3 \mathrm{~d}$, the lymphocyte proliferative response was determined by $\left[{ }^{3} \mathrm{H}\right]$ thymidine incorporation, as described in Methods. Data are expressed as mean ${ }^{3} \mathrm{H}$ incorporation $\pm \mathrm{SD}$. These data are representative of results obtained from three separate experiments. 


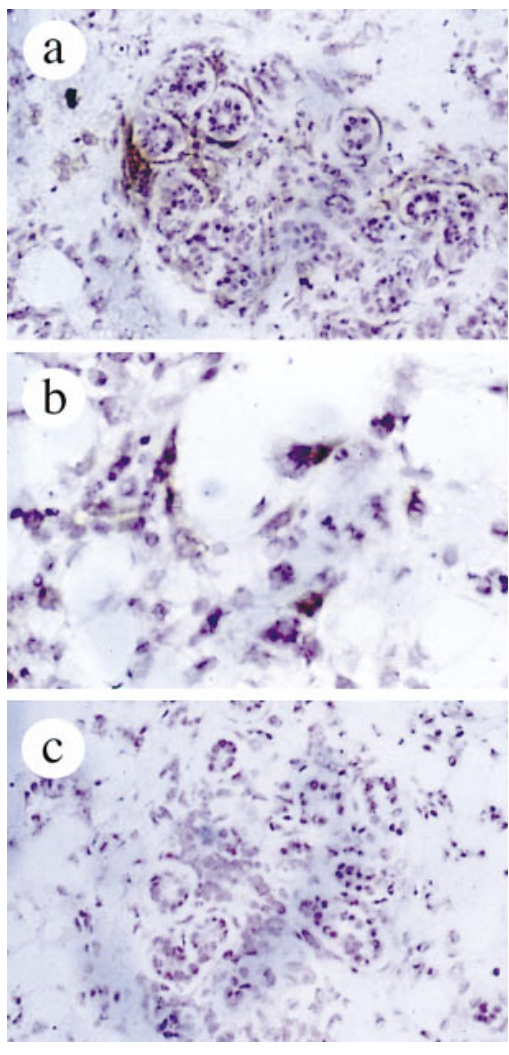

Figure 2. SEB gene expression in tumor tissues after direct DNA injection. Biopsies were obtained from melanomas before treatment and again $48 \mathrm{~h}$ after a single intratumoral injection of SEB plasmid DNA complexed to a cationic lipid. Tumor sections were immunostained with affinitypurified rabbit antiserum to SEB and counterstained with hematoxylin, as described in Methods. Cells positive for SEB protein (brown-black) were focally numerous at several locations throughout the tumor $(a)$. At higher magnification, intracellular staining for SEB was observed in several positive cells within tumor tissues $(b)$. SEB-injected tissues did not react with irrelevant rabbit antiserum $(c)$,

and pretreatment tumor specimens gave minimal background staining with anti-SEB antiserum (data not shown). $a$ and $c, \times 100 ; b$, $\times 200$. line (data not shown). From these data, it was estimated that individual transfected melanoma cells produced between 5.0 and 10.0 fg SEB both intracellularly and extracellularly. This SEB concentration was within the response range (1.0-10.0 $\mathrm{fg} / \mathrm{ml}$ ) of human and canine $\mathrm{T}$ cells (data not shown). Thus, even modest transfection efficiencies in vivo could theoretically generate local concentrations of SEB sufficient to trigger activation and proliferation of tumor-infiltrating lymphocytes.

$S E B$ expression in melanoma tissues after in vivo transfection. SEB expression was detected in three of five tumor specimens after a single SEB DNA injection. Expression was focally intense in some regions of the tumor, and most of the expressed SEB appeared to be intracellular (Fig. 2, $a$ and $b$ ). Although the exact identity of the SEB-positive cells was not determined, it is likely that at least some tumor cells were producing SEB. SEB immunoreactivity was not observed in posttreatment specimens stained with an irrelevant rabbit antiserum (anti-CAT; Fig. $2 c$ ), and SEB antiserum did not stain pretreatment specimens (data not shown). The duration of SEB gene expression was not assessed in these studies, but based on data from other experiments, expression is unlikely to last more than several days in vivo. ${ }^{2}$

Direct intratumoral injection of SEB DNA induces $T$ cell and macrophage infiltration. Pretreatment and 5-7 d postinjection biopsies were obtained from tumors injected either with SEB and GM-CSF DNA, or with empty vector DNA. Each specimen was immunostained with antibodies to CD4, CD8, and CD11c to characterize the magnitude of the T cell and macrophage infiltrate. After histological examination, the inflammatory infiltrate in each specimen was assigned a semiquantitative score of 0 to +4 (no inflammation to severe in-
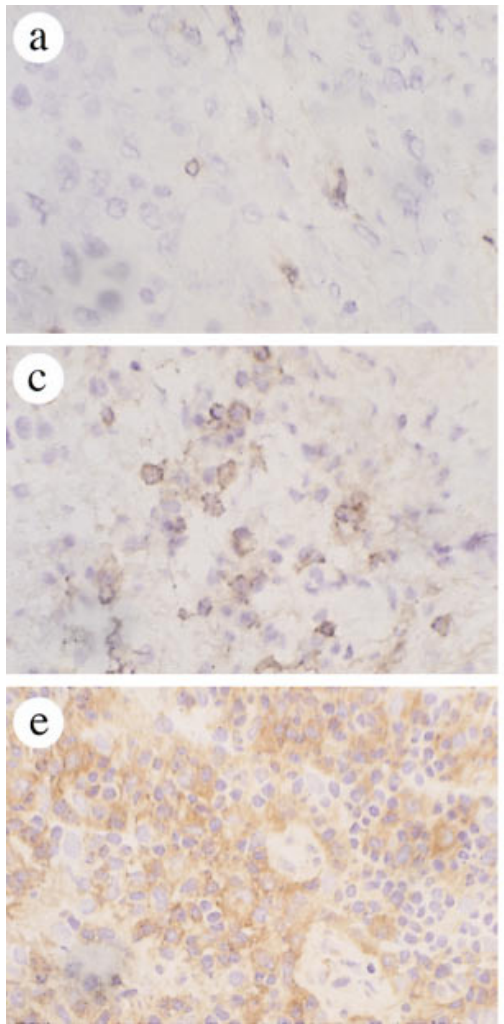
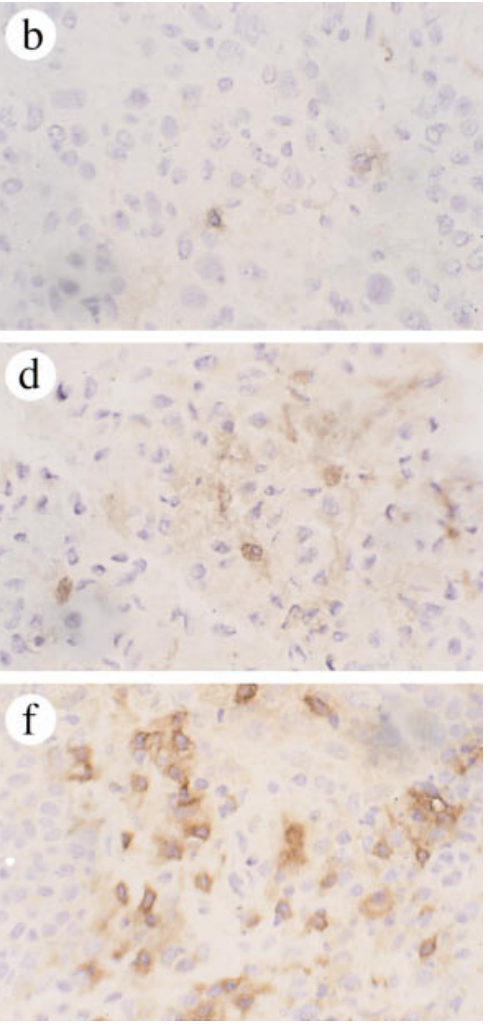

Figure 3. Cellular inflammatory response to intratumoral DNA injections. Immunohistochemistry was used to characterize and compare the intratumoral cellular inflammatory response to direct injection of either SEB plus GM-CSF plasmid DNA (seven biopsies) or empty vector DNA (five biopsies), complexed to cationic liposomes. Tumor biopsies were obtained before injection (seven biopsies) and 5-7 d after injection and immunostained with $\mathrm{mAbs}$ to canine CD4, CD8, and CD11c. Few CD4+ or $\mathrm{CD} 8+\mathrm{T}$ cells were observed in pretreatment melanoma biopsies ( $a$ and $b$ ), although CD11c + cells (macrophages) were relatively numerous in untreated tumors (data not shown). An increase in CD4+ T cells was observed in tumors injected with empty vector DNA $(c)$, whereas CD8+ T cells remained sparse $(d)$. After injection of SEB and GM-CSF DNA, a diffuse infiltrate of CD4+ T cells $(e)$ was observed in most biopsies, along with a large increase in CD8 + T cells $(f)$ and Cd11c + cells (not shown). $a-f$, $\times 165$. 
flammation) in order to compare the effects of injecting empty vector DNA and injecting SEB plus GM-CSF DNA.

Pretreatment biopsies from seven different dogs contained only occasional CD4+ or CD8+ T cells (Fig. 3, $a$ and $b$ ), although $\mathrm{Cd} 11 \mathrm{c}+$ cells (macrophages) were more numerous (mean inflammation scores $=1.0,1.0$, and 2.0, respectively). In tumors injected with liposome-complexed empty vector DNA (five biopsies), the number of CD4+ T cells was increased relative to pretreatment biopsies (Fig. $3 c$ ), whereas there was little change in the number of CD8+ T cells (Fig. $3 d$ ) or macrophages (mean inflammation scores $=1.8,1.2$, and 2.4, respectively). Intratumoral injection of SEB and GM-CSF DNA induced a pronounced increase in $T$ cells and macrophages in most injected tumors (nine biopsies) (Fig. 3, $e$ and $f$ ), with mean inflammation scores of 2.4, 2.6, and 3.6, respectively. Thus, whereas intratumoral injection of empty vector DNA induced mild inflammatory responses (largely consisting of CD4+ T cells), injection of SEB plus GM-CSF DNA induced much stronger inflammatory responses, particularly among CD8+ T cells and macrophages.

Tumor regression. Tumor regression was documented by serial photography in three dogs with oral melanoma. Dog 1 had a stage I melanoma on the lower mandible (Fig. 4). After two DNA injections (week 4), the tumor increased in size but had also begun to depigment. By week 8 , the tumor had regressed completely and did not recur locally or distantly. The tumor site was examined histologically and was free of tumor cells when the dog died of an unrelated malignancy (hepatocellular carcinoma). Dog 2 presented with a stage III melanoma of the mandible with metastasis to the submandibular lymph node. After three DNA injections (week 6), the tumor had decreased $\sim 60 \%$ in size; it regressed completely by week 12 , at which time cytological examination of the submandibular lymph node revealed that it was free of tumor cells, as was the site of the primary tumor. Dog 3 had a stage III melanoma on the lateral maxilla before treatment with SEB plus GMCSF. A treatment response did not occur until 3 mo after completion of the 12 -wk treatment trial in this patient, when complete remission was observed at $28 \mathrm{wk}$.

Local tumor injection induces systemic antitumor CTL activity. Antitumor CTL activity correlates with melanoma regression in both human patients and experimental murine models $(36,37)$. Therefore, antitumor CTL responses were assessed in three dogs whose melanomas had either undergone complete remission (Fig. 5, top left and top right) or partial remission (Fig. 5, top middle), and in three dogs whose tumors were growing progressively (Fig. 5, bottom). In the three dogs with regressing tumors, high levels of antitumor CTL activity were detected in PBMC (Fig. 5, top). Furthermore, in the dogs that achieved complete remission, high CTL activity was sustained for at least 3 mo after the completion of treatment (data not shown). The CTL activity was restricted to autologous tumor cells, as there was minimal lysis of allogeneic melanoma target cells (Fig. 5, top). By contrast, three dogs with progressively growing tumors had minimal CTL reactivity against autologous tumor cells (Fig. 5, bottom). Thus, at least in this small group of animals, the presence of high antitumor CTL activity in PBMC correlated with tumor regression. The temporal appearance of CTL activity was also examined in one dog (Fig. 6). This dog was treated initially with GM-CSF only for $12 \mathrm{wk}$, then switched to a combination of SEB plus GMCSF. Before treatment with the gene combination, there was
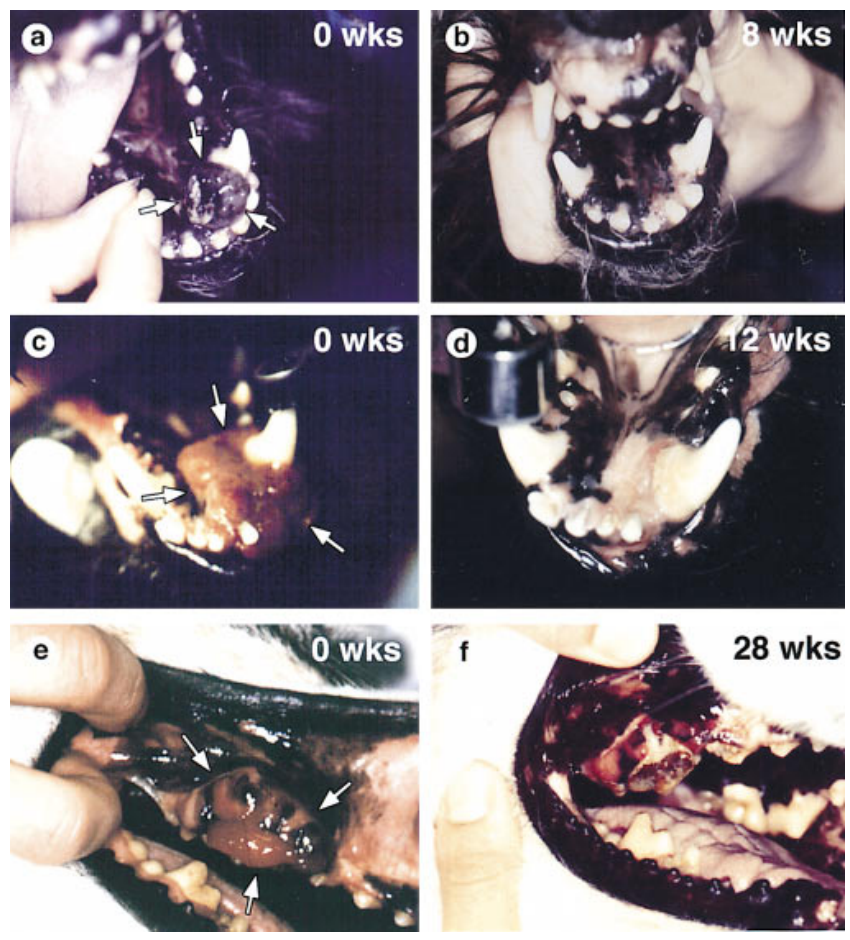

Figure 4. Tumor regression after intratumoral injection. Sequential photographs were used to document tumor regression in three dogs undergoing treatment with SEB and GM-CSF DNA injections. Arrows, Pretreatment tumor dimensions. Dog 1 had a stage I melanoma on the lower mandible (a). After two DNA injections (week 4), the tumor had increased in size but had also begun to depigment. By week 8 , the tumor had regressed completely and did not recur locally or distantly $(b)$. Dog 2 had a stage III melanoma of the rostral mandible with metastasis to the submandibular lymph node $(c)$. After three DNA injections (week 6), the tumor had decreased $\sim 60 \%$ in size. Tumor regression was complete by week $12(d)$. Dog 3 had a stage III melanoma on the lateral maxilla (e). A treatment response did not occur until 3 mo after initiating treatment in this patient, at which time complete regression occurred $(f)$.

no detectable CTL reactivity against the autologous tumor. After the second injection of SEB plus GM-CSF DNA (week 4), CTL activity could be detected, and the level of activity increased further by week 10 (Fig. 6). Tumor regression was first apparent beginning on week 8 . Thus, CTL activity increased with time of treatment and correlated temporally with an objective tumor response.

$P B M C$ proliferative responses to $S E B$. In mice, systemic administration of bacterial SAg proteins may induce deletion and/or anergy of SAg-responsive T cells $(21,38)$. To assess the impact of repeated injections of SEB DNA on T cell responsiveness to $\mathrm{SEB}$, the proliferative response of canine $\mathrm{T}$ cells to purified SEB protein was assessed in vitro, using PBMC obtained from four dogs given at least six injections of SEB plus GM-CSF DNA and from four untreated, age-matched control dogs. The mean stimulation index of treated and control animals was not significantly different, indicating that local intratumoral expression of SEB did not lead to detectable deletion or anergy of SEB-reactive circulating T cells (data not shown).

Clinical responses to treatment. 22 dogs with malignant melanoma were treated with the combination of SEB plus 


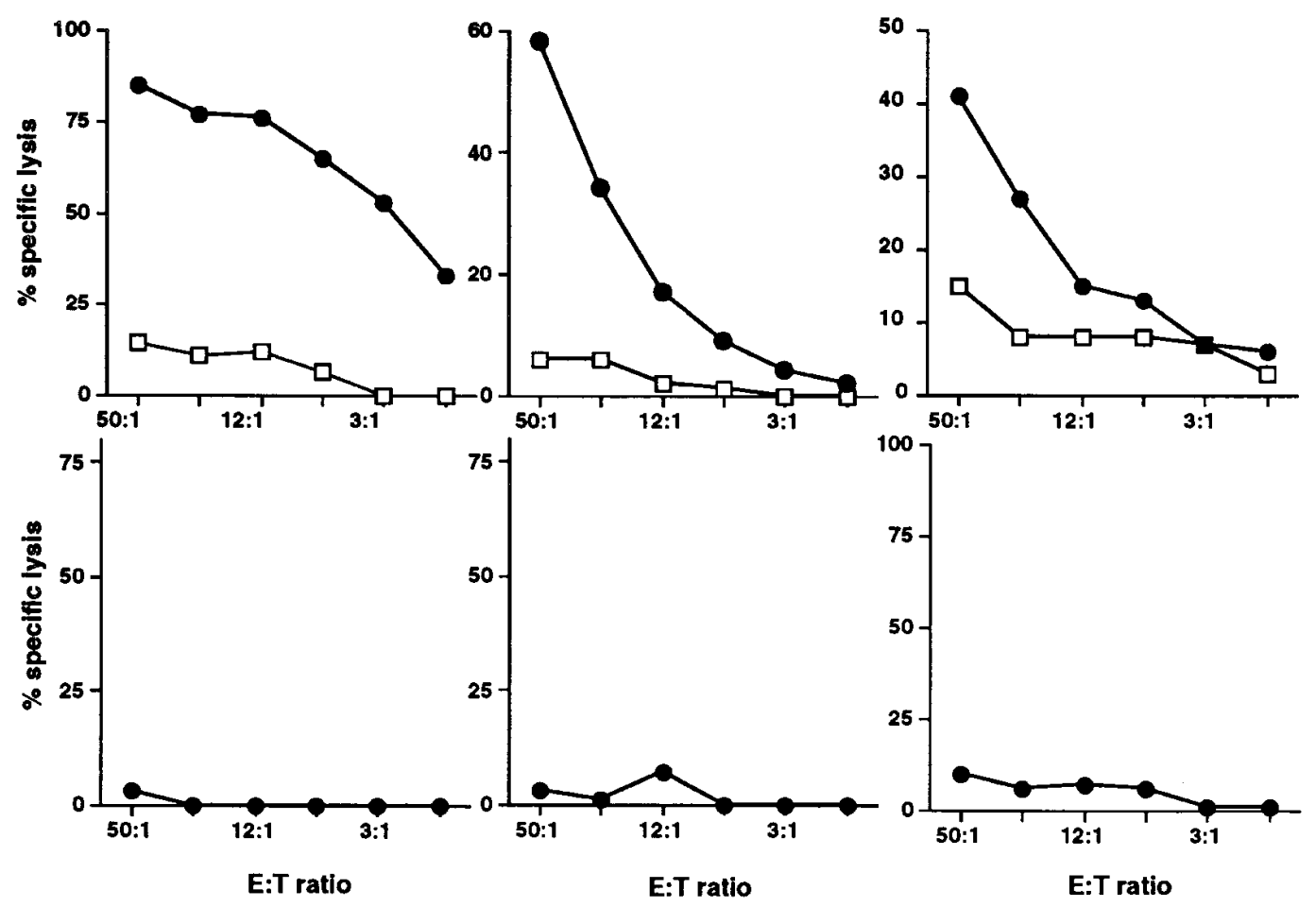

Figure 5. CTL activity in animals with regressing melanomas compared with animals with progressively growing tumors. The antitumor CTL activity in peripheral blood lymphocytes of responding animals (top) was compared with that of animals with progressively growing tumors (bottom), using primary autologous melanoma cell lines. CTL activity was quantitated in PBMC of two dogs whose tumors had undergone complete remission (top right and top left) and one dog whose tumor had undergone partial remission (top middle) after treatment with SEB plus GM-CSF DNA injection. CTL activity was also quantitated in one animal with a rapidly grow-

ing tumor before treatment with SEB and GM-CSF (bottom left) and in two animals with progressively growing tumors that were unresponsive to intratumoral DNA injections (bottom middle and bottom right). Patient PBMC (effectors) were restimulated with irradiated autologous melanoma cells for $6 \mathrm{~d}$, then assayed for cytotoxicity against autologous (filled circles) and allogeneic (open squares) ${ }^{51} \mathrm{Cr}$-labeled melanoma target cells, using a 4-h ${ }^{51} \mathrm{Cr}$ release assay as described in Methods. Data is presented as percentage of specific lysis at decreasing E/T ratios. These assays were repeated at least once for each patient, with similar results.

GM-CSF DNA (Table I). 20 dogs had primary oral malignant melanoma, and 2 had limb lymph node metastasis from digital melanomas. There were 2 dogs with stage IV disease (primary tumor plus radiographically visible lung metastases), 12 patients with stage III tumors (primary tumor $4.0 \mathrm{~cm}$ or greater in the longest dimension, plus lymph node spread), 5 dogs with stage II tumors (tumor $2.0-4.0 \mathrm{~cm}$, lymph nodes not involved), and 3 dogs with stage I tumors (tumors $<2.0 \mathrm{~cm}$ ) (32). Of the 22 dogs enrolled, 16 survived to complete the 12-wk initial treatment trial. All but one of the six dogs that did not complete the 12-wk initial trial had very advanced disease (stage III or IV), and all six died within $10 \mathrm{wk}$ of entering the study.

The overall response rate (complete or partial remission) for all 22 dogs enrolled in this study was 41\% (Table I). All 3 dogs with stage I disease had a treatment response (partial or complete remission), whereas $60 \%$ (3 of 5) with stage II disease responded, and 33\% (4 of 12) with stage III disease responded. Neither of the two dogs with stage IV disease responded to treatment. Five dogs were treated initially with single gene therapy (three with SEB alone and two with GMCSF alone), but none responded, and all were switched to combination gene therapy.

In responding animals, a decrease in tumor size usually occurred within $10 \mathrm{wk}$ (mean $7 \mathrm{wk}$ ) of the initiation of treatment. One dog had a delayed response, with no tumor regression observed until 2 mo after treatment. Tumor necrosis or evidence of immediate tumor cytotoxicity as a result of DNA injection was not observed in any treated patients. Tumor depigmenta- tion occurred in one tumor during tumor regression (Fig. 4), but evidence of cutaneous depigmentation in normal tissues was not observed in any treated dogs. None of the dogs with complete remission of their primary tumors had local recurrence. Tumor size was a major determinant of tumor responses in this study. The response rate for stage I and II tumors was 100 and $60 \%$, respectively, whereas the response rate was $33 \%$ for stage III tumors and $0 \%$ for stage IV tumors (Table I).

Four dogs with stage III melanoma were treated with SEB plus canine IL-2 DNA in a pilot study that followed the original SEB plus GM-CSF study. This second pilot study was initiated based on data from experimental tumor studies in mice that indicated a significant augmentation of the SAg antitumor effect by coinjection of SAg plus IL-2. ${ }^{2}$ There was a $75 \%$ tumor response rate (three of four dogs with complete tumor remissions) at $12 \mathrm{wk}$ in dogs treated with SEB plus IL-2. The nonresponding dog was killed at 5 mo after entry. One responding dog was killed due to lung metastases at 6 mo after treatment, while another responding dog died of an unrelated disease at 15 mo after treatment (on complete necropsy, there was no detectable melanoma). The fourth responding dog remained tumor-free at 18 mo after treatment.

Treatment-related toxicity was minimal in dogs in this study, and fever was not observed at any time in any patient. One dog developed transient peritumoral edema after injection on two separate occasions, and a second patient experienced two separate episodes of transient anorexia ( $24 \mathrm{~h}$ duration). There were no significant changes in complete blood 


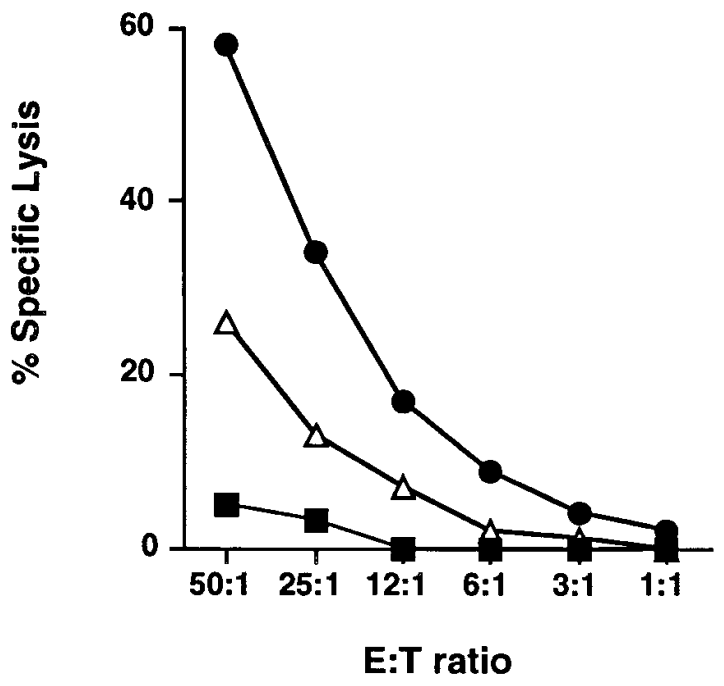

Figure 6. Development of increasing CTL activity over time after direct intratumoral treatment. CTL activity was evaluated sequentially in a dog with malignant melanoma treated by intratumoral SEB plus GM-CSF DNA injections. At the start of treatment, the tumor was growing progressively, and CTL activity was minimal (squares). After 4 wk of treatment with SEB plus GM-CSF DNA, CTL activity could be detected in PBMC (triangles), and the level of CTL activity increased further by week 10 of treatment (circles), at which time partial tumor regression had occurred.

counts or serum biochemical parameters during or at the completion of the study in dogs treated with either liposome and DNA formulation (data not shown). Thus, local expression of an SAg gene did not induce systemic toxicity or organ dysfunction in any treated patients, despite that fact that dogs are fully susceptible to the adverse effects of SAgs administered systemically $(39,40)$.

Table I. Clinical Response and Survival Data from 22 Dogs with Melanoma Treated by Direct Intratumoral Injection of SEB plus GM-CSF DNA

\begin{tabular}{lcccc}
\hline Tumor stage & No. of patients & Response & $\begin{array}{c}\text { Median } \\
\text { survival }\end{array}$ & $\begin{array}{c}\text { Mean } \\
\text { survival }\end{array}$ \\
\hline & & $\%$ & $w k$ & $w k$ \\
I & 3 & 100 & 61 & 61 \\
II & 5 & 60 & 57 & 47 \\
III & 12 & 33 & 24 & 42 \\
IV & 2 & 0 & ND & 7 \\
& & & &
\end{tabular}

Canine melanoma patients were staged according to World Health Organization guidelines for tumors of domestic animals, using the TNM system (32). Stage I tumors measured $<2 \mathrm{~cm}$ in their greatest dimension; stage II tumors measured between 2 and $4 \mathrm{~cm}$ in greatest dimension; stage III tumors measured $>4 \mathrm{~cm}$ in greatest dimension, and/or lymph node metastasis was present; dogs with stage IV tumors had radiographically detectable lung metastases. Animals were treated with twice-monthly injections of equal amounts of SEB and GM-CSF DNA complexed to a cationic lipid, as described in Methods. The primary tumor was measured twice monthly for $12 \mathrm{wk}$, then monthly thereafter. A positive treatment response was recorded for any animal whose tumor underwent either complete or partial $(>50 \%$ reduction in tumor volume) remission during or after treatment. $N D$, Not done.
Survival times. A previous study of dogs with malignant melanoma found that stage III melanoma patients treated by surgery alone (excision of the primary tumor and any affected lymph nodes) developed metastatic disease and did not survive beyond $22 \mathrm{wk}$, with a median survival time of $15 \mathrm{wk}$ (41). By contrast, survival times for stage III melanoma dogs in this study treated by intratumoral injection of SEB plus GM-CSF were prolonged significantly $(P<0.02)$ compared with the historical control patients (Fig. 7). Five dogs survived to $56 \mathrm{wk}$, and four dogs survived to at least $90 \mathrm{wk}$ after treatment (Fig. 7). The median survival time for nine stage III dogs that survived to complete the 12 -wk induction phase of treatment was $66 \mathrm{wk}$. Thus, local intratumoral immunotherapy increased survival times significantly, presumably as a consequence of generating systemic antitumor immunity sufficient to eradicate pulmonary micrometastases. 11 of $16 \mathrm{dogs}$ (all tumor stages) that survived beyond the first 12 wk of treatment eventually died (Table I). However, not all dogs died of melanoma: three died of unrelated diseases (no evidence of local or metastatic melanoma at necropsy), whereas eight died from melanoma metastases to the lungs.

Detection of antibodies to SEB. Serum of 10 untreated control dogs and 8 dogs treated with the combination of SEB plus GM-CSF-direct DNA injection was assayed for IgG antibodies to SEB. Serum was obtained from treated patients at time points from 10 to $20 \mathrm{wk}$ after entry into the study. Antibodies to SEB were not detected in the serum of any untreated control animals (data not shown). Antibodies to SEB were

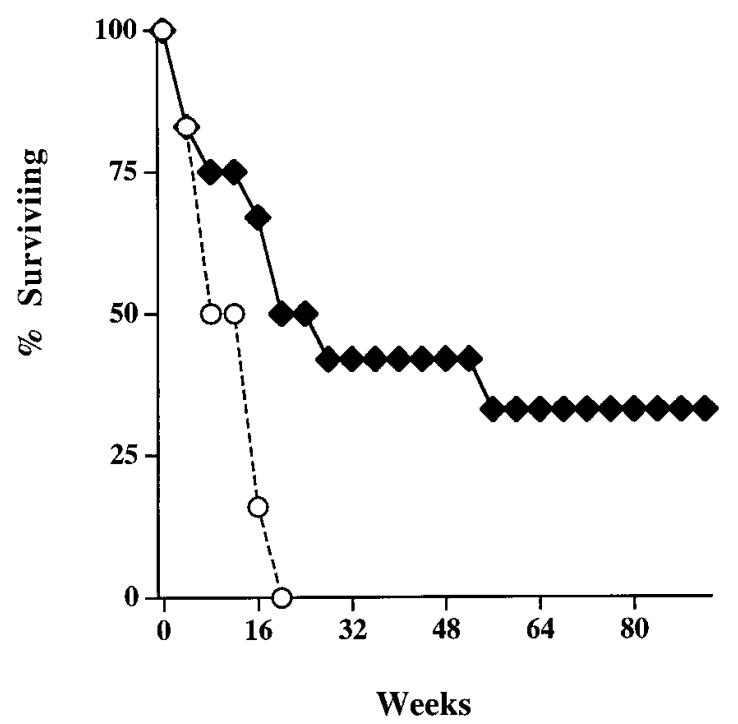

Figure 7. Survival times in dogs with stage III melanoma: surgically treated controls versus intratumoral gene therapy patients. Survival times for eight control dogs (circles) with stage III melanoma that had been treated by complete surgical excision of the primary tumor and lymph node metastases (reference 41; data used with permission of Dr. E.G. MacEwen, University of Wisconsin) were plotted, together with survival times for 12 dogs (diamonds) with stage III melanoma that were treated only with intratumoral injections of SEB plus GM-CSF DNA. The survival time for SEB plus GM-CSF DNAtreated dogs was significantly $(P<0.02)$ longer than for control dogs. Four of the gene-treated dogs remained alive at $90 \mathrm{wk}$ after treatment, whereas all control dogs treated with surgery alone were dead by 21 wk. 
present in the serum of four of eight treated animals, in titers that ranged from 1:4,000 to 1:512,000. The presence of an SEBspecific response was confirmed in these patients by Western blotting (data not shown).

\section{Discussion}

We found in this study that intratumoral expression of an SAg gene and a cytokine gene induced both local and systemic antitumor immune responses against established, spontaneously arising malignant melanomas. Development of local antitumor immunity was demonstrated by intratumoral infiltration of $\mathrm{T}$ cells and macrophages and by tumor regression, whereas development of systemic antitumor immunity was evidenced by CTL activity in peripheral blood lymphocytes and by significantly increased survival times. These results are important inasmuch as they are to our knowledge the first demonstration of the ability of local intratumoral gene therapy in a spontaneous tumor model to induce complete and sustained local regression of large tumor burdens, as well as to generate significantly prolonged, disease-free survival times.

The effectiveness of this approach to tumor immunotherapy may depend to a large degree on the strong T cell-stimulatory properties of bacterial SAgs. The primary role of the coinjected cytokine gene is probably to modify and/or enhance the effects induced by the SAg gene. The GM-CSF gene was used together with the SEB gene in this study based on the ability of GM-CSF to stimulate macrophages and dendritic cells and to enhance antigen presentation to T cells $(1,5)$. The IL-2 gene, when coexpressed locally with an SAg, may act to increase the local survival and function of tumor-infiltrating lymphocytes attracted and activated in situ by the locally expressed SAg. This cytokine enhancement has been observed in experimental tumor studies in mice when the IL-2 gene was combined with the SAg gene. ${ }^{2}$ In addition, repeated injections may also be important to generate sufficient $T$ cell activation to regress large tumor burdens, particularly in light of recent studies by Speiser et al. (18), who found that multiple immunizations were necessary to overcome the state of partial tolerance that develops to tumor antigens in animals with established autologous tumors.

Intratumoral expression of SEB DNA did not induce clinically important toxicity in treated dogs. Thus, the local concentrations of SEB produced in tumor tissues were too low to induce systemic toxicity, but were sufficient to induce local T cell migration and activation. Furthermore, abnormalities ostensibly linked to the chronic effects of SAgs (e.g., immune-mediated diseases) have not been observed in any treated dogs during observation periods of up to $2 \mathrm{yr}$. Systemic administration of SAg proteins to mice may induce T cell deletion and/or anergy $(21,38)$. However, McCormack et al. (42) demonstrated that coadministration of a specific antigen along with a bacterial SAg rescued the antigen-responsive $\mathrm{T}$ cells from SAginduced deletion. Thus, tumor-specific $\mathrm{T}$ cells infiltrating into tumor tissues may be resistant to SAg-induced deletion in vivo. Furthermore, peripheral deletion and/or anergy of SEBreactive $T$ cells were not observed in treated dogs.

Bacterial DNA is immunogenic in animals, and studies have demonstrated a nonspecific antitumor effect from intratumoral injections of noncoding plasmid DNA complexed to a cationic lipid $(43,44)$. Nonetheless, studies in mice have indicated that intratumoral injections of SAg and cytokine DNA were significantly more effective than empty vector alone against established melanomas. ${ }^{2}$ In addition, immunohistochemical analysis of DNA-injected canine tumors revealed a more pronounced CD8 $+\mathrm{T}$ cell and macrophage infiltrate in tumors injected with SEB plus GM-CSF compared with tumors injected only with empty vector DNA (Fig. 3).

Like melanoma in humans, the prognosis for canine melanoma correlates directly with tumor stage, and melanoma in dogs is refractory to chemotherapy and irradiation and also does not respond well to treatment with conventional biological response modifiers such as Mycobacterium bovis BCG or Corynebacterium parvum extracts $(31,32,41)$. Survival time is an important measure of the efficacy of immunotherapeutics for melanoma, since most melanoma patients ultimately die of metastatic disease. Thus, it is noteworthy that survival times for SAg plus cytokine-treated dogs with stage III melanoma were significantly longer than those of historical control animals. Furthermore, the SAg-cytokine-treated group also included a number of long-term surviving animals (Fig. 8). Given the more rapid progression of canine melanoma and the shorter life-span of dogs relative to humans, these results assume added significance. Lipid-mediated DNA delivery may also be used to target metastatic tumors in noncutaneous sites such as the lung and liver. Studies are currently under way to evaluate the effectiveness of these alternative DNA-targeting approaches for metastatic cancer.

\section{Acknowledgments}

The authors wish to acknowledge the excellent technical assistance of Angela Sandusky, Wendy Woolridge, Dr. Melissa McPherron, and Mr. David Kummer, the photographic assistance of Ms. Leigh Landskroner, and the secretarial assistance of Ms. Kathy Morgan. In addition, the authors thank Dr. Peter Moore for use of the canine leukocyte antibodies, and Dr. Steve Radecki for assistance with statistical analysis.

This work was supported in part by a grant from the Colorado Cancer League, and by Megabios Corporation, Heska Corporation, and Veterinary Cancer Specialists.

\section{References}

1. Pardoll, D.M. 1993. New strategies for enhancing the immunogenicity of tumors. Curr. Opin. Immunol. 5:719-725.

2. Fearon, E.R., D.M. Pardoll, T. Itaya, P. Golumbek, H.I. Levitsky, J.W. Simons, H. Karasuyama, B. Vogelstein, and P. Frost. 1990. Interleukin-2 production by tumor cells bypasses $\mathrm{T}$ helper function in the generation of an antitumor response. Cell. 60:397-403.

3. Gansbacher, B., K. Zier, B. Daniels, K. Cronin, R. Bannerji, and E. Gilboa. 1990. Interleukin-2 gene transfer into tumor cells abrogates tumorigenicity and induces protective immunity. J. Exp. Med. 172:1217-1224.

4. Golumbek, P.T., A.J. Lazenby, H.I. Levitsky, L.M. Jaffee, H. Karasuyama, M. Baker, and D.M. Pardoll. 1991. Treatment of established renal cell cancer by tumor cells engineered to secrete interleukin-4. Science. 254:713-716.

5. Dranoff, G., E. Jaffee, A. Lazenby, P. Golumbek, H. Levitsky, K. Brose, V. Jackson, H. Hamada, D. Pardoll, and R. Mulligan. 1993. Vaccination with irradiated tumor cells engineered to secrete granulocyte-macrophage colonystimulating factor stimulates potent, specific, and long-lasting anti-tumor immunity. Proc. Natl. Acad. Sci. USA. 90:3539-3543.

6. Lotze, M.T. 1996. Cytokine gene therapy of cancer. Cancer J. 2:63-72.

7. Townsend, H.E., and J.P. Allison. 1993. Tumor rejection after direct costimulation of CD8+ T cells by B7-transfected melanoma cells. Science. 259: 368-370.

8. Li, Y., P. McGowan, I. Hellstrom, K.E. Hellstrom, and L. Chen. 1994. Costimulation of tumor-reactive CD4+ and CD8+ T lymphocytes by B7, a natural ligand for CD28, can be used to treat established mouse melanoma. J. Immunol. 153:421-427.

9. Baskar, B.S., L. Glimcher, N. Nabavi, R.T. Jones, and S. RosenbergOstrand. 1995. Major histocompatibility complex class II+B7-1+ tumor cells 
are potent vaccines for stimulating tumor rejection in tumor-bearing mice. $J$. Exp. Med. 181:619-629.

10. Eynde, B., and V.G. Brichard. 1995. New tumor antigens recognized by T cells. Curr. Opin. Immunol. 7:674-681.

11. Van der Bruggen, P., C. Traversari, P. Chomez, C. Lurquin, E. DePlaen, B. Van den Eynde, A. Knuth, and T. Boon. 1991. A gene encoding an antigen recognized by cytolytic T lymphocytes on a human melanoma. Science. 254: 1643-1647.

12. Mandelboim, O., E. Vadam, M. Fridkin, A. Katz-Hillel, M. Feldman, G. Berke, and L. Eisenbach. 1995. Regression of established murine carcinoma metastases following vaccination with tumour-associated antigen peptides. Nat. Med. 1:1179-1183.

13. Young, J.W., and K. Inaba. 1996. Dendritic cells as adjuvants for class I major histocompatibility complex-restricted antitumor immunity. J. Exp. Med. 183:7-11.

14. Zitvogel, L., J.L. Mayordomo, T. Tjandrawan, A.B. DeLeo, M.R. Clarke, M.T. Lotze, and W.J. Storkus. 1996. Therapy of murine tumors with tumor peptide-pulsed dendritic cells: dependence on T cells, B7 costimulation, and T helper cell 1-associated cytokines. J. Exp. Med. 183:87-97.

15. Boczkowski, D., S.K. Nair, D. Snyder, and E. Gilboa. 1996. Dendritic cells pulsed with RNA are potent antigen-presenting cells in vitro and in vivo. $J$. Exp. Med. 184:465-472.

16. Plautz, G.E., Z.Y. Yang, B.Y. Wu, X. Gao, L. Huang, and G.J. Nabel. 1993. Immunotherapy of malignancy by in vivo gene transfer into tumors. Proc. Natl. Acad. Sci. USA. 90:4645-4649.

17. Nabel, G.J., E.G. Nabel, Z.Y. Yang, B.A. Fox, G.E. Plautz, X. Gao, L. Huang, S. Shu, D. Gordon, and A.E. Chang. 1993. Direct gene transfer with DNA-liposome complexes in melanoma: expression, biologic activity, and lack of toxicity in humans. Proc. Natl. Acad. Sci. USA. 90:11307-11311.

18. Speiser, D.E., R. Miranda, A. Zazkarian, M.F. Bachmann, K. McKallFaienza, B. Odermatt, D. Hanahan, R.M. Zinkernagel, and P.S. Ohashi. 1997. Self-antigens expressed by solid tumors do not efficiently stimulate naive or activated T cells: implications for immunotherapy. J. Exp. Med. 186:645-653.

19. Rakmilevich, A.L., J. Turner, M.J. Ford, D. McCabe, W.H. Sun, P.M. Sondel, K. Grota, and N. Yang. 1996. Gene gun-mediated skin transfection with interleukin-12 gene results in regression of established primary and metastatic murine tumors. Proc. Natl. Acad. Sci. USA. 93:6291-6296.

20. Sun, W.H., J.K. Burkholder, J. Sun, J. Culp, J. Turner, X.G. Lu, T.D. Pugh, W.B. Ershler, and N. Yang. 1995. In vivo cytokine gene transfer by gene gun reduces tumor growth in mice. Proc. Natl. Acad. Sci. USA. 92:2889-2893.

21. Kotzin, B.L., D.Y.M. Leung, J. Kappler, and P. Marrack. 1993. Superantigens and their potential role in human disease. Adv. Immunol. 54:99-165.

22. Leung, D.Y.M., G.M. Gately, A. Trumble, B. Ferguson-Darnell, P.M. Schlievert, and L.J. Picker. 1995. Bacterial superantigens induce T cell expression of the skin-selective homing receptor, the cutaneous lymphocyte-associated antigen, via stimulation of interleukin-12 production. J. Exp. Med. 181: $747-753$

23. Sriskandan, S., T.J. Evans, and J. Cohen. 1996. Bacterial superantigeninduced human lymphocyte responses are nitric oxide dependent and mediated by IL-12 and IFN-gamma. J. Immunol. 156:2430-2435.

24. Lando, P.A., G. Hedlund, M. Dohlsten, and T. Kalland. 1991. Bacterial superantigens as anti-tumour agents: induction of tumour cytotoxicity in human lymphocytes by staphylococcal enterotoxin A. Cancer Immunol. Immunother. 33:231-237.

25. Ochi, A., K. Migita, J. Xu, and K. Simonovitch. 1993. In vivo tumor immunotherapy by a bacterial superantigen. J. Immunol. 151:3180-3186.

26. Newell, K.A., J.D.I. Ellenhorn, D.S. Bruce, and J.A. Bluestone. 1991. In vivo T-cell activation by staphylococcal enterotoxin B prevents outgrowth of a malignant tumor. Proc. Natl. Acad. Sci. USA. 88:1074-1078.
27. Shu, S., R.A. Krinock, T. Matsumura, J.L. Sussman, B.A. Fox, A.E. Chang, and D.S. Terman. 1994. Stimulation of tumor-draining lymph node cells with superantigenic staphylococcal toxins leads to the generation of tumor-specific effector cells. J. Immunol. 152:1277-1288.

28. Dohlsten, M., L. Abrahamsen, P. Bjork, P.A. Lando, G. Hedlund, G. Forsberg, T. Brodin, N.R.J. Gascoigne, C. Forberg, P. Lind, and T. Kalland. 1994. Monoclonal antibody-superantigen fusion proteins: tumor-specific agents for T-cell-based tumor therapy. Proc. Natl. Acad. Sci. USA. 91:8945-8949.

29. Dow, S.W., and T.A. Potter. 1997. Expression of bacterial superantigen genes in mice induces localized mononuclear cell responses. J. Clin. Invest. 99: 2616-2623.

30. Carlsson, R., H. Fischer, and H.O. Sjogren. 1988. Binding of staphylococcal enterotoxin A to accessory cells is a requirement for its ability to activate human T cells. J. Immunol. 140:2484-2488.

31. Harvey, H.J., E.G. MacEwen, D. Braun, and A.K. Patnaik. 1981. Prognostic criteria for dogs with oral melanoma. J. Am. Vet. Med. Assoc. 178:580-582.

32. Withrow, S.J. 1989. Tumors of the gastrointestinal system and oral cavity. In Clinical Veterinary Oncology. S.J. Withrow and E.G. MacEwen, editors. J.B. Lippincott Co., Philadelphia. 227-261.

33. Zhu, N., D. Liggitt, Y. Liu, and R. Debs. 1993. Systemic gene expression after intravenous DNA delivery into adult mice. Science. 261:209-211.

34. Solodin, I., C.S. Brown, M.S. Bruno, C. Chow, E. Jang, R.J. Debs, and T.D. Heath. 1995. A novel series of amphiphilic imidazolinium compounds for in vitro and in vivo gene delivery. Biochemistry. 34:13537-13544.

35. Cobbold, S.P., and S. Metcalf. 1994. Monoclonal antibodies that define canine homologues of human CD antigens: summary of the first international canine leukocyte antigen workshop (CLAW). Tissue Antigens. 43:137-154.

36. Kawakami, Y., S. Eliyahu, C. Jennings, K. Sakaguchi, X. Kang, S. Southwood, P.F. Robbins, A. Sette, E. Appella, and S.A. Rosenberg. 1995. Recognition of multiple epitopes in the human melanoma antigen gp100 by tumor infiltrating lymphocytes associated with in vivo tumor regression. J. Immunol. 154:3961-3968

37. Rosenberg, S.A., B.S. Packard, B.M. Aebersold, D. Solomon, S.L. Topalian, S.T. Toy, P. Simon, M.T. Lotze, J.C. Yang, C.A. Seipp, et al. 1988. Use of tumor-infiltrating lymphocytes and interleukin-2 in the immunotherapy of patients with metastatic melanoma. A preliminary report. N. Engl. J. Med. 319: $1676-1680$.

38. Herman, A., J.W. Kappler, P. Marrack, and A.M. Pullen. 1991. Superantigens: mechanism of T-cell stimulation and role in immune responses. Annu. Rev. Immunol. 9:745-772.

39. Prohaska, J.V., V. Kocandrle, and E. Houttuin. 1966. Hemodynamic changes in response to staphylococcal exotoxin and enterotoxin. Bull. Soc. Int. Chir. 25:727-736.

40. Gilbert, C.F. 1966. Effects of staphylococcal enterotoxin B on the coagulation mechanism and leukocytic response in beagle dogs: a preliminary study. Thromb. Diath. Haemorrh. 16:697-706.

41. MacEwen, E.G., A.K. Patnaik, H.J. Harvey, A.A. Hayes, and R. Matus. 1986. Canine oral melanoma: comparison of surgery versus surgery plus Corynebacterium parvum. Cancer Invest. 4:397-406.

42. McCormack, J.E., J. Kappler, and P. Marrack. 1994. Stimulation with specific antigen can block superantigen-mediated deletion of $\mathrm{T}$ cells in vivo. Proc. Natl. Acad. Sci. USA. 91:2086-2090.

43. Pisetsky, D.S. 1996. Immune activation by bacterial DNA: a new genetic code. Immunity. 5:303-310.

44. Parker, S.E., S. Khatibi, M. Margalith, D. Anderson, M. Yankauckas, S.H. Gromkowski, T. Latimer, D. Lew, M. Marquet, M. Manthorpe, et al. 1996. Plasmid DNA gene therapy: studies with the human interleukin-2 gene in tumor cells in vitro and in the murine B16 melanoma model in vivo. Cancer Gene Ther. 3:175-185. 